\title{
TECHNIQUES D'ADRESAGE DE MATRICES À CRISTAUX LIQUIDES
}

\author{
T. LEROUX
}

LETI, Division du Commissariat a 1 'Energie Atomique,

CENG/LETI/DOPT/SCMM, BP. 85X, F-38041 Grenoble Cedex, France

\section{I - PREAMBULE}

La structure matricielle (voir fig.1) est, pour des raisons que nous développerons, à la base de toutes les technologies d'écrans plats. Appliquée au domaine des cristaux liquides, elle induit des contraintes qui, évidemment, limitent la marge de manoeuvre des fabricants et suscitent un effort de recherche important visant à en réduire les effets, mais doivent être également prises en compte par les utilisateurs s'ils veulent optimiser le fonctionnement de ces composants.

Parmi les technologies les plus répandues et les plus étudiées :

- les technologies des écrans multiplexés classiques utilisant principalement des cristaux liquides en phase nématiques et au nombre desquelles on trouve les technologies TN, STN (Twisted et Super Twisted Nematic) et BCE (Birefringence Contrôlée Electriquement),

- les technologies utilisant des matrices actives (de dipôles non linéaires ou de transistors) qui visent à réduire les limitations des écrans à multiplexage direct,

- la technologie plus récente des cristaux liquides ferroélectriques (smectiques C chiraux) qui tire parti des propriétés de bistabilité et de vitesse de cette nouvelle famille de cristaux liquides,

nous n'étudierons en détail que les 2 premieres, la dernière faisant l'objet d'un cours spécifique ( $\mathrm{Cf}$ ce volume).

Nous supposerons par ailleurs connues, les propriétés de base des cristaux liquides et les technologies de fabrication des cellules pour nous concentrer sur les problèmes posés à l'utilisateur par l'adressage des écrans plats matriciels. 


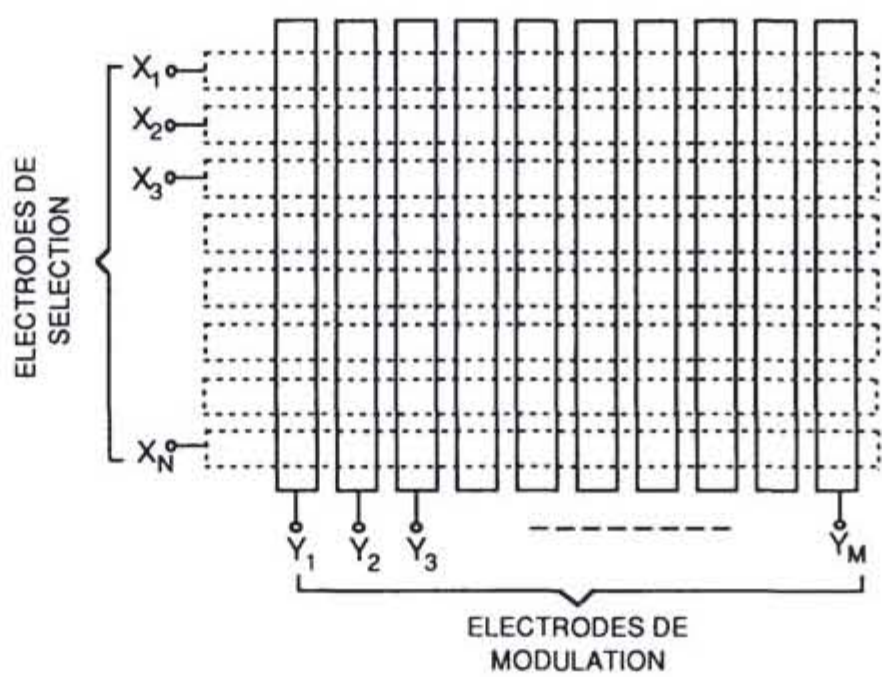

Fig. 1 - Structure matricielle
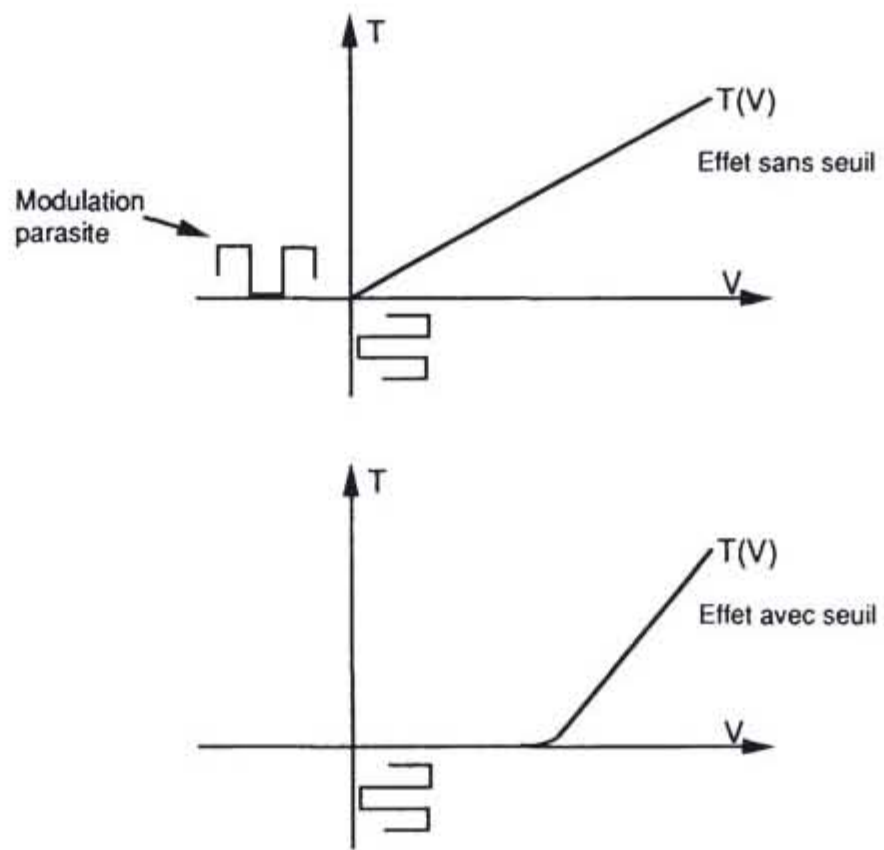

Fig. $2 a$ et $2 b$ - Influence des signaux de modulation 


\section{II - L'ORGANISATION MATRICIELLE, AVANTAGES ET CONTRAINTES}

L'utilisation de cette structure se justifie par la réduction importante du nombre de connexions nécessaires à l'adressage qu'elle permet. En effet, comme on peut le voir figure 1, l'adressage de $M \times N$ points élémentaires (pixels) peut être réalisé grace à $M+N$ connexions.

Les lignes 1 à $\mathrm{N}$ sont sélectionnées séquentiellement, les colonnes 1 à $\mathrm{M}$ recevant lors de ces sélections des signaux propres au contrôle des pixels de la ligne considérée. Cette méthode, le multiplexage, induit certaines contraintes, ainsi :

- Si l'effet utilisé nécessite une durée TI pour l'adressage d'une ligne, le nombre maximal de lignes adressables en un temps trame Tt (usuellement $20 \mathrm{~ms}$ ) sera de $\mathrm{Tt} / \mathrm{Tl}$.

- L'effet utilisé doit posséder un seuil (voir figures 2a et 2b). En effet les pixels des lignes non adressées subissent constamment les signaux colonnes destinés à d'autres lignes. II est visible (figure 2a) que si l'effet n'a pas de seuil, ces signaux induiront sur les pixels une modulation parasite source de couplage (influence du reste de l'image sur un pixel considéré) et de baisse de contraste. Un effet à seuil ne présente pas cet inconvénient (figure $2 \mathrm{~b}$ ).

Ce dernier problème peut être pris en compte par l'adressage. Ainsi, le mode dit V/3V ( voir figure 3) où :

- les signaux lignes sont à $+\frac{2 \mathrm{~V}}{3}$ lorsqu'elles sont adressées et 0 dans le cas contraire.

- les signaux colonnes sont à $-V / 3$ pour les pixels sélectionnés et $+V / 3$ sinon.

permet de garder fixe quelque soit la situation considérée le rapport Amplitude appliquée à un pixel sélectionné (V)/Amplitude appliquée à un pixel non sélectionné (V/3). Nous verrons cependant qu'un tel mode adapté plus particulièrement aux écrans utilisant des effets symétriques (indépendants du signe des tensions appliquées) et actifs uniquement lors d'une sélection, comme par exemple les écrans à matrice active utilisant des dipoles non linéaires, n'est pas optimal pour une matrice à cristaux liquides à multiplexage direct.

\section{III - MATRICES A MULTIPLEXAGE DIRECT}

\section{a) Structure et propriétés}

La structure matricielle d'écrans à cristaux liquides la plus naturelle (figure 4) consiste en une cellule ou lignes et colonnes sont placées sur chacun des substrats la constituant, le cristal liquide $(C L)$ étant inséré entre celles ci. Les $C L$ utilisés le plus souvent appartiennent, si l'on exclut le cas des écrans à C.L. ferroélectriques, à la catégorie des CL nématiques. Les méthodes employées pour orienter les molécules de ceux-ci dans la cellule et obtenir un effet électrooptique utilisable permettent de distinguer les différentes technologies (TN, STN, BCE,...). Le comportement des cellules du point de vue de l'utilisateur reste néanmoins peu dépendant de celles-ci, ainsi :

- Les pixels peuvent, étant donné la très forte résistivité des mélanges CL utilisés, être modelisés par des capacités. 


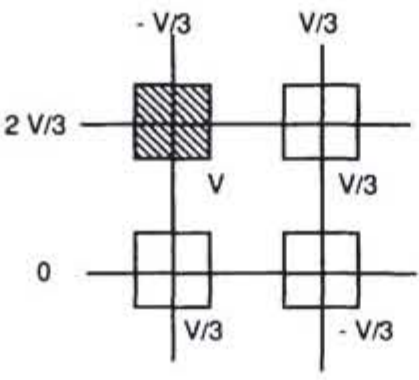

Pixel sélectionné

Fig. 3 - Tensions appliquées dans le mode d'adressage "V/3V"

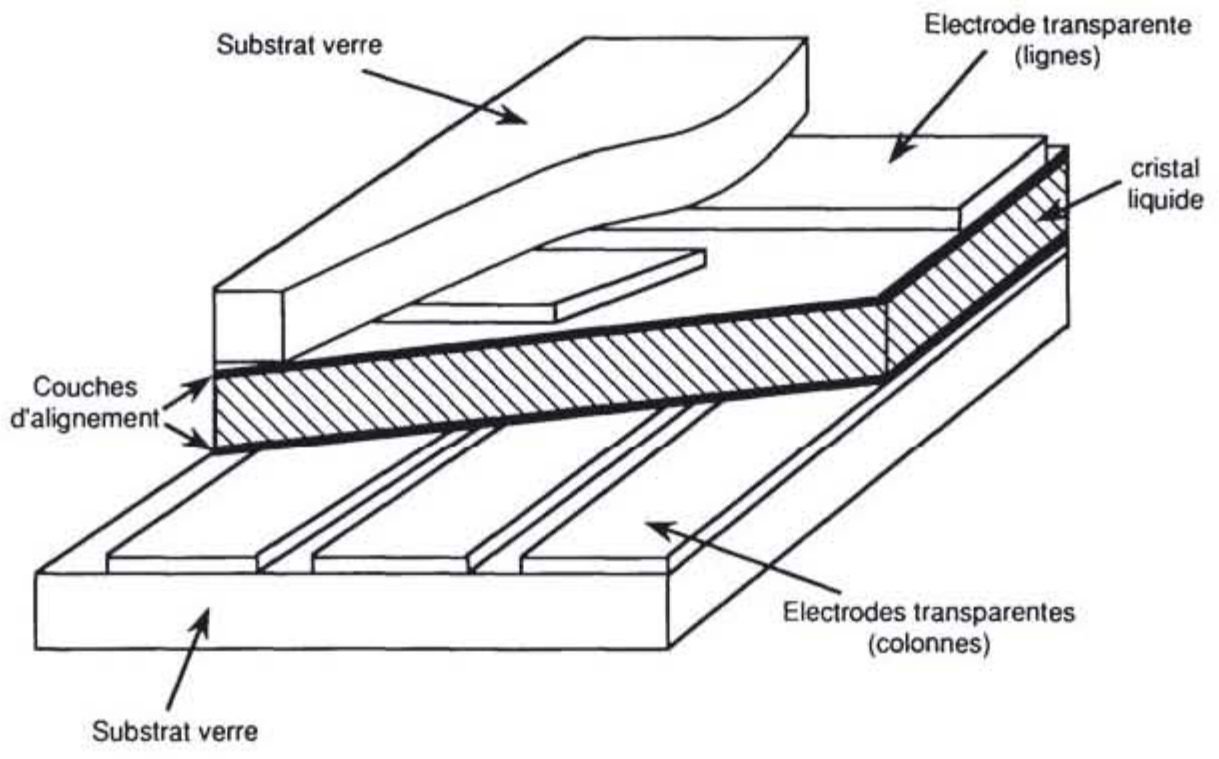

Fig. 4 - Vue éclatée d'une cellule à CL à multiplexage direct 


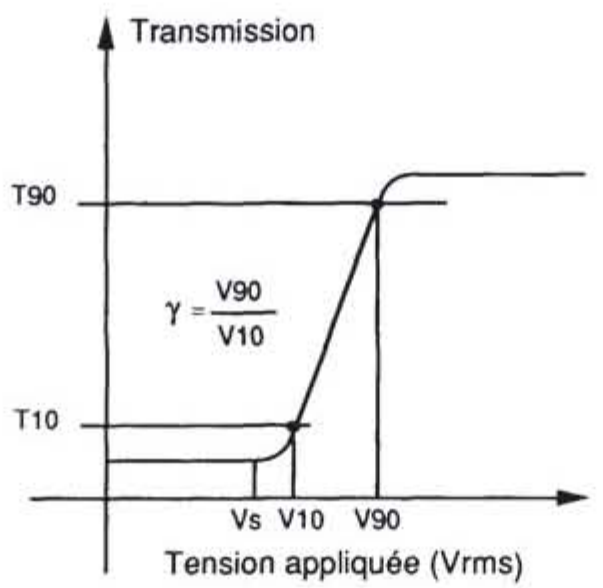

Fig. 5 - Réponse électrooptique d'une cellule à cristal liquide

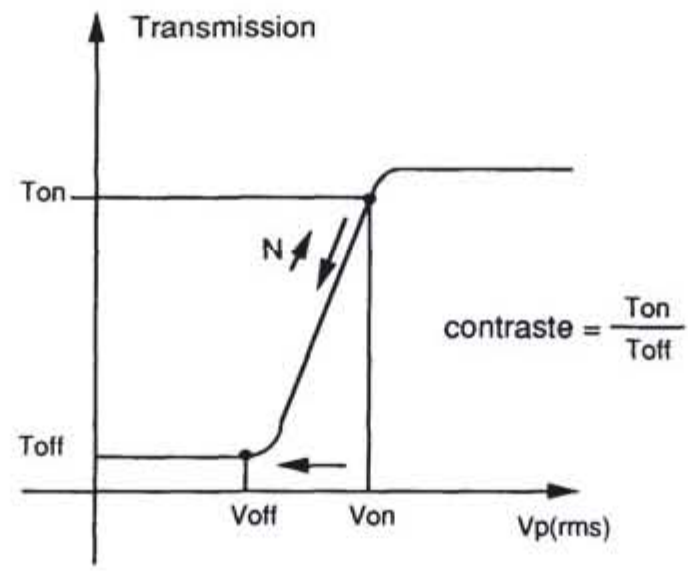

Fig. 6 - Points de fonctionnement d'un écran multiplexé ; influence du nombre de lignes adressées 

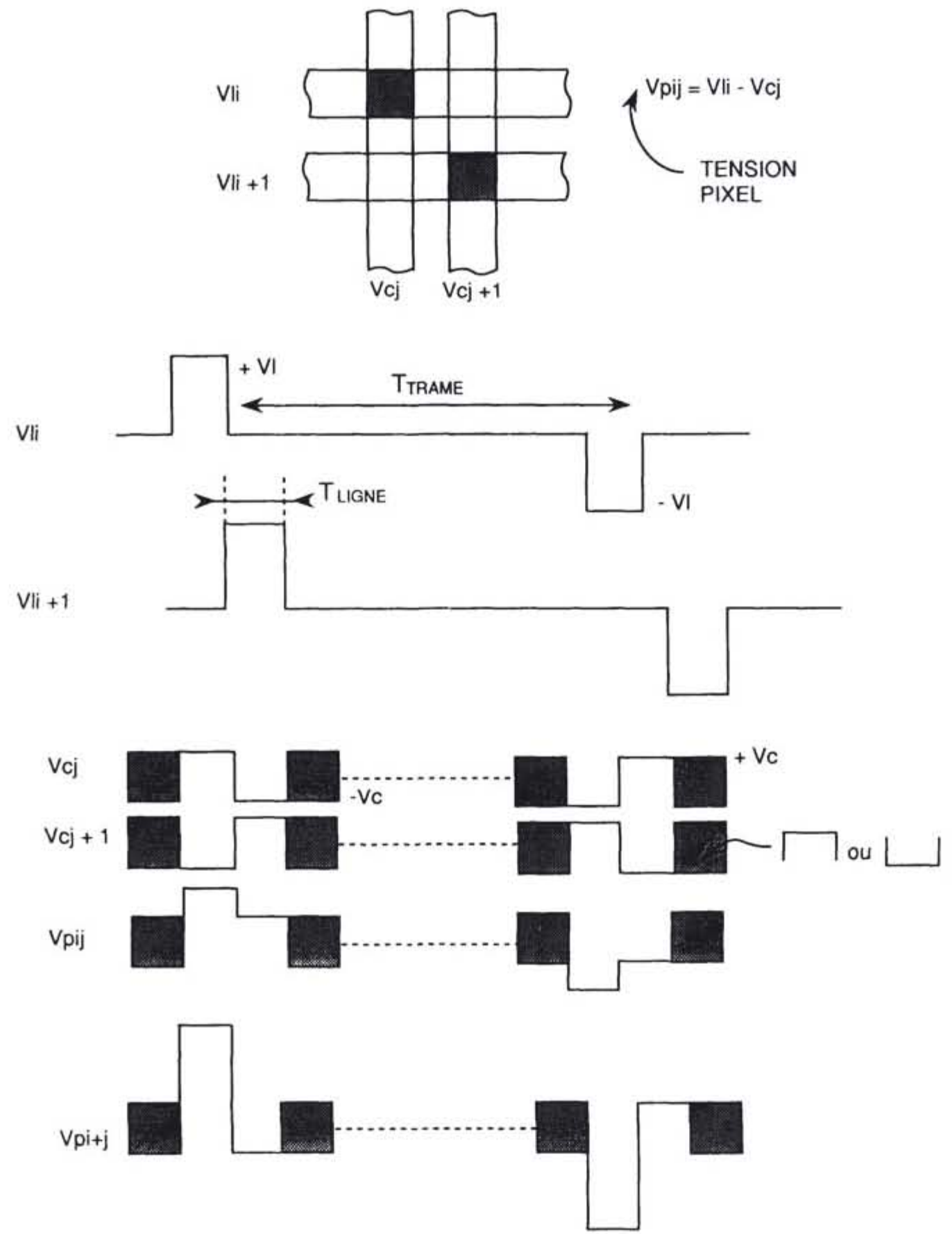

Fig. 7 - Multiplexage direct d'une matrice 
- L'utilisateur devra veiller à ce que les signaux appliqués aux lignes et aux colonnes soient de valeur moyenne nulle. En effet, toute présence d'une composante continue (>50 m V) entraine une électrolyse du CL et une dégradation des performances de la matrice.

- La courbe de réponse électrooptique prend l'allure indiquée figure 5.

On relève sur cette dernière les particularités suivantes :

- La cellule répond à la valeur efficace de la tension appliquée (Vrms). Cette propriété très importante du point de vue de l'adressage des matrices peut être comprise qualitativement si l'on considère que d'une part de $\mathrm{CL}$ est un milieu visqueux donc intégrateur et que d'autre part, pour les $C L$ nématiques, les molécules peuvent êtres considérées comme porteuses d'un dipole induit $(P=E)$. En conséquence,les forces appliquées aux molécules sont proportionnelles au carré du champ appliqué $\left(F=P E \approx E^{2}\right)$ et la transmission fonction de :

$\frac{1}{T} \int_{0}^{T} v^{2} d t$

Donc de la valeur efficace de la tension appliquée.

- La courbe T (V) présente un seuil Vs. Le seuil assure la mutiplexabilité des matrices et peut s'exprimer en fonction de paramètres physiques du CL par :

$V s=\Pi \sqrt{\frac{k}{\varepsilon 0 \Delta \varepsilon}}$

oủ $K$ est une fonction des constantes élastiques du mélange utilisé et $\Delta \varepsilon$ son anisotropie dielectrique. On remarquera que ce seuil ne dépend pas, en première approximation, de l'épaisseur de la cellule utilisée.

\section{b) Adressage, principe de base}

La figure 7 présente le principe utilisé pour l'adressage des écrans à multiplexage direct :

- Une impulsion d'amplitude VI (+VI pour la trame $\mathrm{K}$, - VI pour la trame K+1 de façon à respecter $\langle V\rangle=0$ ) permet de sélectionner successivement les lignes de la matrice.

- Un signal + Vc ou - Vc (inversé à chaque trame) appliqué aux colonnes permet en se retranchant ou en s'ajoutant à la tension appliquée sur la ligne de moduler la transmission des pixels. On remarquera que linformation (signaux colonnes) est codée par la polarité des signaux et non par leur amplitude.

De façon plus détaillée, la tension $\mathrm{Vp}$ appliquée à un pixel peut s'exprimer par :

$$
V p^{2}=\frac{1}{N}(V I \pm V c)^{2}+\frac{N-1}{N} V_{c}^{2}
$$

oủ $\mathrm{N}$ est le nombre de lignes de l'écran.

On constate que le terme $\frac{\mathrm{N}-1}{\mathrm{~N}} \mathrm{Vc}^{2}=\mathrm{Vc}^{2}$, provenant des signaux colonnes destinés aux autres lignes, est constant et indépendant du contenu de l'image affichée, ce qui garantit l'absence de couplage. Ce terme constitue par ailleurs une "polarisation" du point de 
fonctionnement des pixels à laquelle s'ajoute le terme $\frac{1}{N}(V L \pm V c)^{2}$ qui selon la polaritè du signal colonne " décide" de l'état du pixel.

\section{c) Optimisation des tensions appliquées}

Le contraste entre pixels sélectionnés et non sélectionnés rest le paramètre le plus important pour nombre d'applications. II sera d'autant meilleur que l'on saura maximiser le rapport $\frac{\text { Von }}{\text { Voff }}$ où :

$$
\begin{aligned}
& V_{o n^{2}}=\frac{(V I+V c)^{2}}{N}+\frac{N-1}{N} V c^{2} \\
& V o f f 2=\frac{(V I-V c)^{2}}{N}+\frac{N-1}{N} V c^{2}
\end{aligned}
$$

expriment les tensions appliquées respectivement aux pixels sélectionnés et non sélectionnés.

$$
\begin{aligned}
& \text { On pose } V 1=V I+V c \text { et } b=\frac{V 1}{V c} \text { d'où : } \\
& V_{0 n^{2}}=\frac{V 1^{2}}{N}+\frac{(N-1)}{N}\left(\frac{V 1}{b}\right)^{2} \\
& V_{o f f}{ }^{2}=\frac{V 1^{2}}{N}\left(\frac{b-2}{b}\right)^{2}+\frac{N-1}{N}\left(\frac{V 1}{b}\right)^{2} \\
& \text { et }\left(\frac{V \text { on }}{V \text { off }}\right)^{2}=\frac{b^{2}+N-1}{(b-2)^{2}+N-1}
\end{aligned}
$$

La maximisation de $\left(\frac{\text { Von }}{\text { Voff }}\right)^{2}$ conduit à $: b=1+\sqrt{ } \mathrm{N}$

d'oú $\frac{\text { Von }}{\text { Voff }}=\sqrt{\frac{\sqrt{N}+1}{\sqrt{N}-1}}=1+\frac{1}{\sqrt{N}}$ pour $N$ grand

Le rapport Von/Voff optimal peut donc être exprimé en fonction du seul nombre de lignes et tend vers 1 comme $1 / \sqrt{ } \mathrm{N}$ lorsque $\mathrm{N}$ est grand (voir figure 8 ). II s'en suit que pour une réponse électrooptique donnée, le contraste obtenu sera d'autant plus faible que le nombre de lignes de la matrice sera important (voir figure 6).

Le paramètre important est ici la raideur du seuil électrooptique $\gamma$ :

$$
\gamma=\frac{V 90}{V 10}
$$

ou V90 et $\mathrm{V} 10$ sont les tensions nécessaires pour obtenir $90 \%$ et $10 \%$ de transmission. En effet dans la région du seuil :

$$
T=T \min +\frac{(T \max -T \min )}{(V \max -V s)}(V-V s)
$$




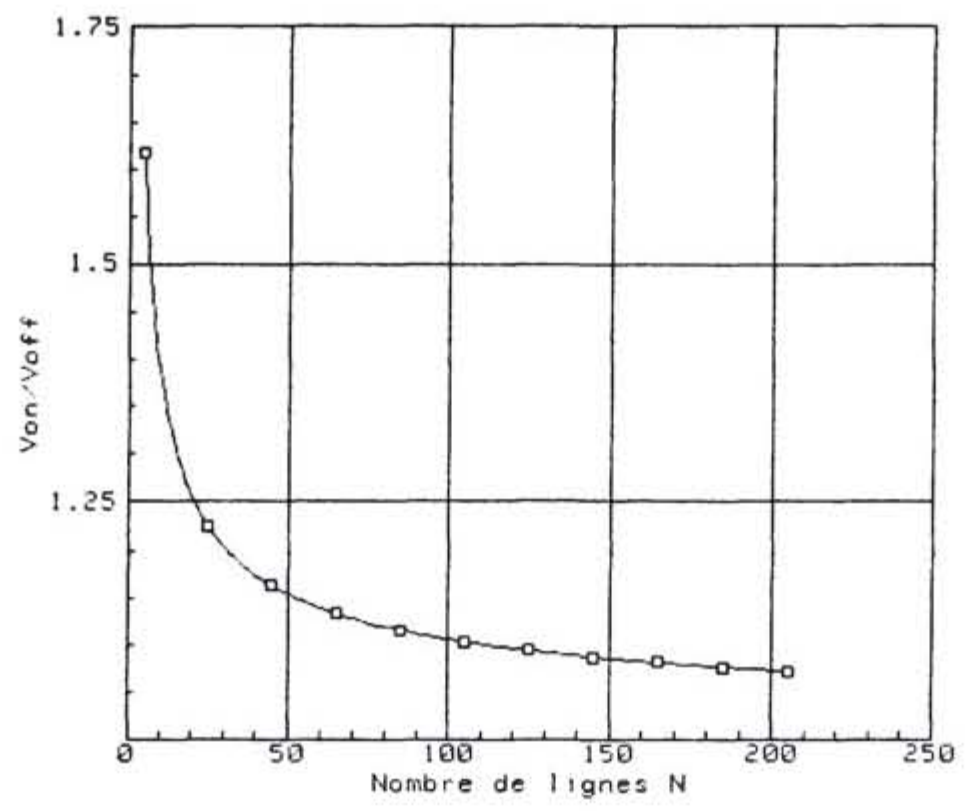

Fig. 8 - Courbe Von/Voff optimal en fonction de $\mathrm{N}$

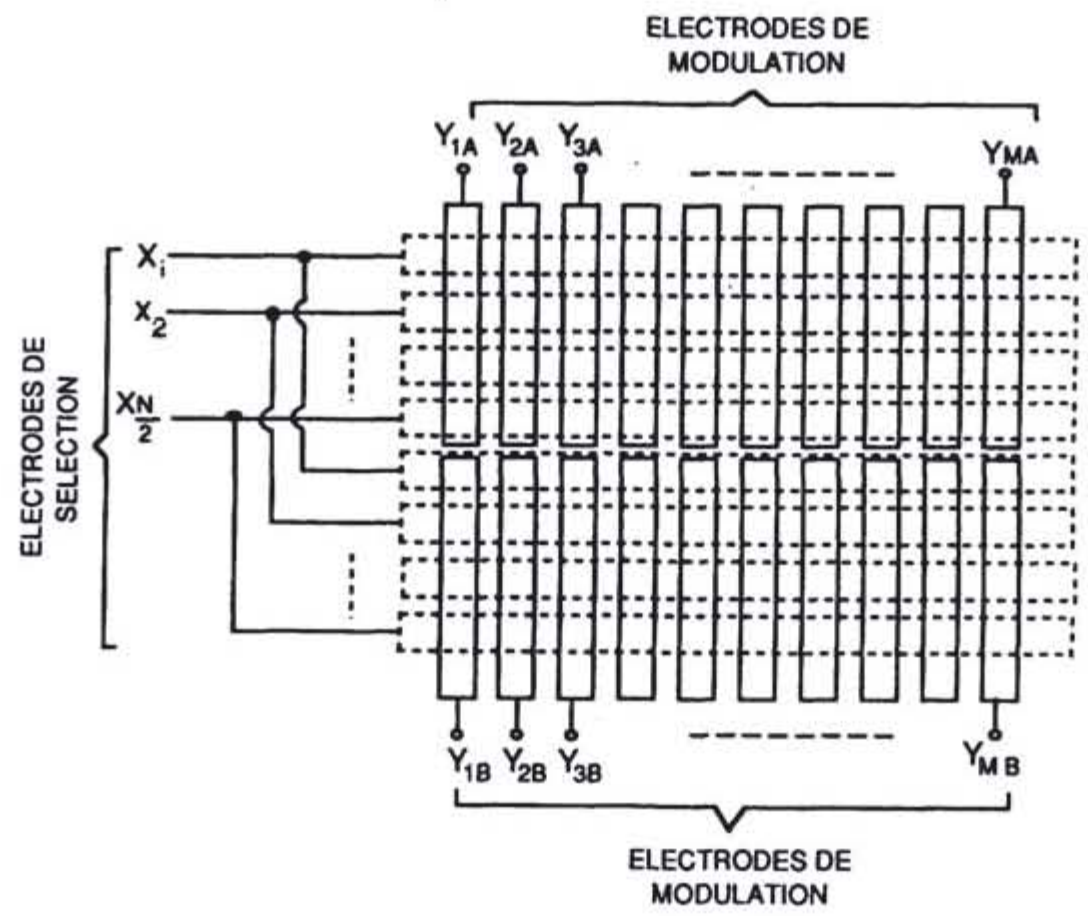

Fig. 9 - Structure double matrice 


$$
\begin{aligned}
& \text { or } C=\frac{\text { Ton }}{\text { Toff }} \text { avec Voff }=V s \text { donc Toff } \approx T \text { min } \\
& \text { ainsi } C=1+\frac{(\text { Tmax }- \text { Toff })}{(\text { Vmax }- \text { Voff })} \frac{(\text { Von }- \text { Voff })}{\text { Toff }} \\
& \text { d'où } C=1+\frac{\text { CMAX }}{\sqrt{N}(\gamma-1)}
\end{aligned}
$$

Comme on peut le voir le contraste c décroit comme $1 / \sqrt{ } \mathrm{N}$ et sera d'autant meilleur que $\gamma$ sera proche de 1.

\section{d) Réalisation pratique de l'adressage}

Les tensions nécessaires à l'adressage d'une matrice peuvent être obtenues à partir des expressions précédentes. En effet :

$$
\begin{aligned}
& \text { Voff }=\frac{V 1 \sqrt{2}}{\sqrt{N}+1}\left[1-\frac{1}{\sqrt{N}}\right]^{1 / 2} \\
& =V 1 \sqrt{2} / \sqrt{N} \text { si } N \text { grand } \\
& =V s \\
& \text { d'oủ } V 1=\sqrt{\frac{N}{2}} V s=V I+V c \text { et } \frac{V 1}{V c}=b=\sqrt{N} \\
& \text { donc } V c=\frac{V s}{\sqrt{2}} \text { et } V I=\sqrt{N} V_{c}
\end{aligned}
$$

à titre d'exemple, on dispose à l'heure actuelle de CL permettant d'adresser 240 lignes avec un contraste acceptable $(=10$ à 20 ) et un seuil $\approx 2$ à 2,5 V. Des tensions lignes de 30 à $35 \mathrm{~V}$ sont donc nécessaires, 40 à $50 \mathrm{~V}$ devraient être appliquées pour adresser un écran de 480 lignes à la norme VGA.

C'est pourquoi de tels écrans sont habituellement séparés en 2 demi écrans (voir figure 9) permettant ainsi un gain de $\sqrt{2}$ sur les tensions lignes nécessaires et le contraste obtenu à niveau technologique donné.

Dans le mode d'adressage décrit figure 7,4 alimentations asservies $\pm V l$ et $\pm V c$ sont nécessaires. Les circuits de commande des matrices (drivers) doivent par ailleurs, pour ce qui est des lignes, commuter 3 tensions $+\mathrm{VI}, 0$ et - VI et donc supporter $2 \mathrm{VL}$ à leur bornes. En pratique, ces contraintes conduisent à commuter la référence des tensions entre les trames positives et négatives. Pour ce faire, on ajoute respectivement $+V_{c}$ et $+\mathrm{VI}$ aux tensions requises au cours de ces trames (voir figure 10). 5 tensions V1 à Vs sont alors nécessaires :

$$
\begin{aligned}
& \mathrm{V}_{1}=\mathrm{VI}+\mathrm{Vc} \\
& \mathrm{V}_{2}=\mathrm{VI}_{1}=\left(1-\frac{1}{\mathrm{~b}}\right) \mathrm{V}_{1} \\
& \mathrm{~V}_{3}=\mathrm{VI}-\mathrm{V}_{\mathrm{c}}=\left(1-\frac{2}{\mathrm{~b}}\right) \mathrm{V}_{1}
\end{aligned}
$$



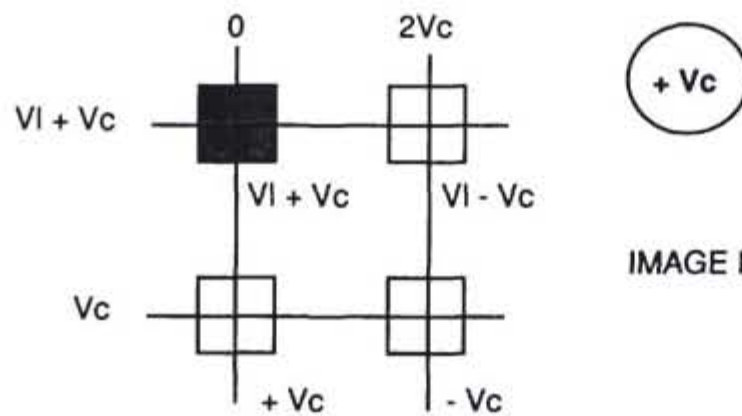

IMAGE K
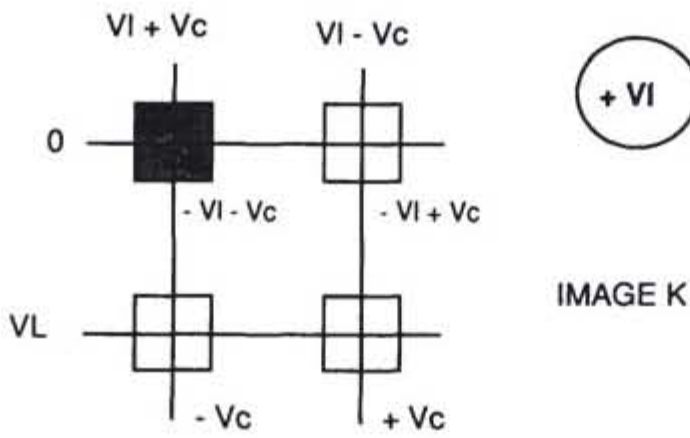

$$
+\mathrm{VI}
$$

IMAGE K + 1

Fig. 10 - Tension appliquée en pratique

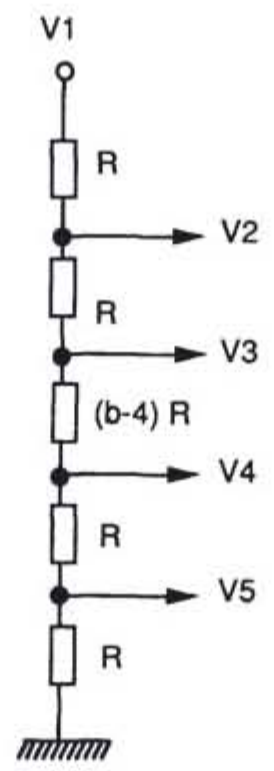

Fig. 11 - Obtention des tensions V1 à V5 à partir d'une alimentation unique 


$$
\begin{aligned}
& \mathrm{V} 4=2 \mathrm{~V}_{\mathrm{c}}=\frac{2}{\mathrm{~b}} \mathrm{~V} 1 \\
& \mathrm{~V} 5=\mathrm{Vc}=\frac{1}{\mathrm{~b}} \mathrm{~V} 1 \\
& \text { avec } \mathrm{V} 1=\sqrt{\frac{\mathrm{N}}{2}} \mathrm{Vs} \text { et } \mathrm{b}=1+\sqrt{\mathrm{N}}
\end{aligned}
$$

Les circuits ne doivent plus désormais supporter que $\mathrm{VI}+\mathrm{Vc} \approx \mathrm{VI}$ et les tensions requises sont très simplement obtenues à partir d'une alimentation unique et d'un pont diviseur résistif (figure 11). Ces tensions sont appliquées aux électrodes en respectant la logique suivante :

\section{Tensions lignes}

Polarité

Ligne

sélectionnée

\begin{tabular}{c|c|c} 
& + & - \\
\hline oui & V1 & 0 \\
\hline non & V5 & V2
\end{tabular}

\section{Tensions colonnes}

Polarité

Pixei

sélectionné

\begin{tabular}{c|c|c} 
& + & - \\
\hline oui & 0 & V1 \\
\hline non & V4 & V3
\end{tabular}

Pour de plus amples informations sur la structure interne des drivers qui, à notre avis, sort du cadre de cet exposé, le lecteur pourra se reporter aux fiches techniques des constructeurs tels que SEIKO, OKI, HITACHI, ... II suffit de savoir que ces circuits se conforment tous au mode d'adressage décrit ci-dessus et mettent à la disposition de l'utilisateur une interface permettant la liaison de l'écran avec l'électronique externe.

\section{e) Niveaux de gris et couleur}

La couleur est obtenu "très simplement" par synthèse additive de l'information de 3 sous pixels rouge vert et bleu par pixel (figure 12). Ces filtres (généralement des polymères colorés) sont placés à l'intérieur de la cellule et déposés par des techniques de photolithographie, électrodéposition ou offset. On rencontre diverses organisations des sous pixels, en colonnes, en diagonale ou en delta plus particulièrement adaptées à l'affichage de textes, d'images vidéo ou polyvalente (figure 13). Si ce n'est qu'elle augmente le nombre de connexions nécessaires et dégrade la transmission de la cellule (d'un facteur > à 3). L'introduction de la couleur n'impose donc à l'utilisateur que le souci d'acheminer correctement l'information afin d'obtenir le résultat escompté.

Pour ce qui est des niveaux de gris, l'obtention d'une transmission intermédiaire entre Ton et Toff (blanc et noir) impose de pouvoir déplacer le point de fonctionnement des pixels 


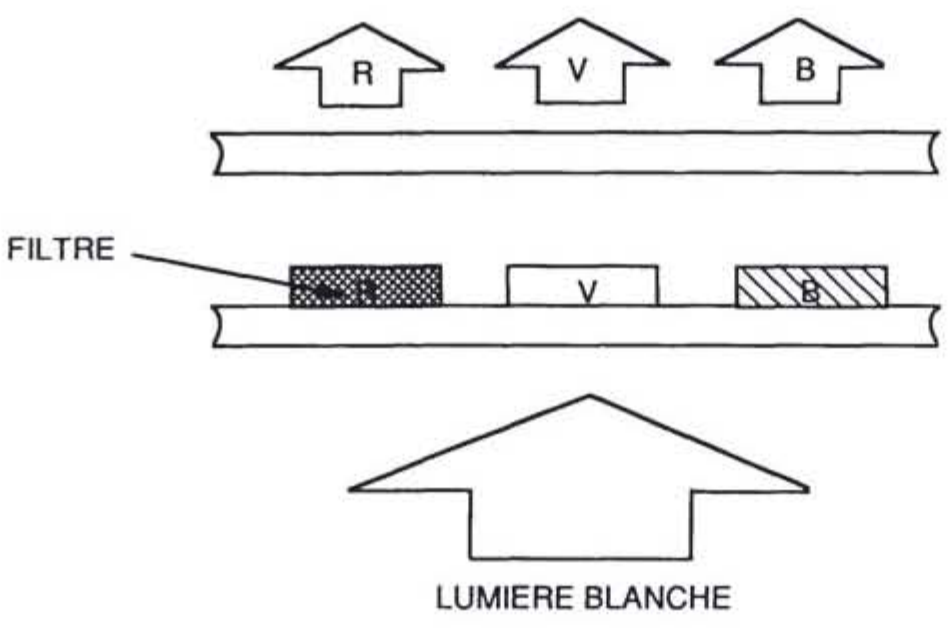

Fig. 12. - Réalisation de la couleur par synthèse additive de 3 sous pixels rouge, vert et bleu

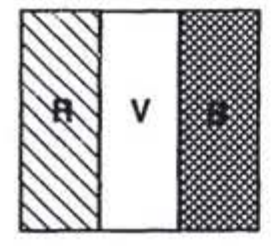

EN COLONNES

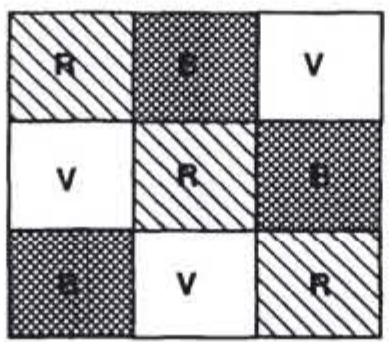

DIAGONALE

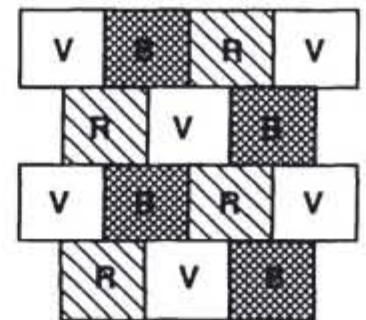

DELTA

Fig. 13 - Distribution les plus courantes des sous pixels dans la matrice 


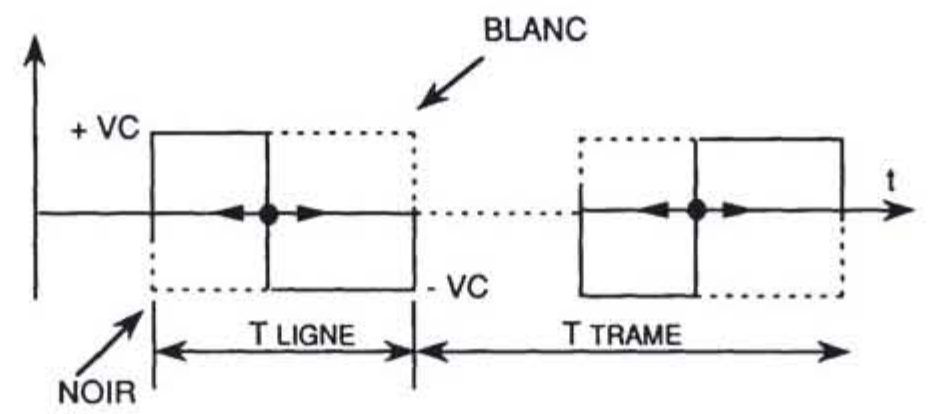

Fig. 14 - Signaux appliqués aux colonnes pour obtenir des niveaux de gris par modulation temporelle

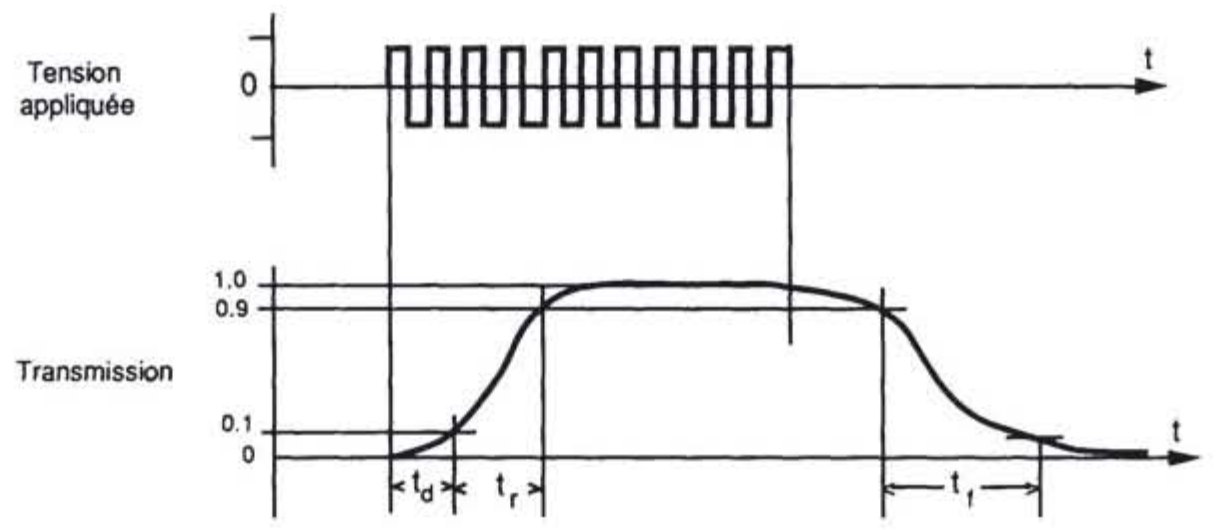

Fig. 15 - Comportement dynamique d'une cellule à cristal liquide 
entre Von et Voff tout en gardant constante et indépendante du contenu de limage l'expression $\sum_{1}^{N} \frac{V_{c i}^{2}}{N}$. Ceci ne peut être obtenu que par modulation temporelle des signaux colonnes (figure 14 ) qui ne prennent ainsi que les valeurs $\pm \mathrm{Vc}$. Les tensions nécessaires sont identiques au cas "Noir et Blanc", un traitement numérique des signaux appliqués aux colonnes permettant de réaliser la modulation temporelle.Des drivers colonnes fournissant 16 niveaux codés sur 4 bits sont actuellement disponibles sur le marché (OKI MSM $5300, \ldots)$. On notera enfin que ,l'obtention de niveaux de gris uniformes sur toute la matrice impose un contrôle précis de la reproductibilité de la caractéristique $T(V)$. Ce contrôle est d'autant plus délicat que le seuil est raide ( $\gamma$ proche de 1) et l'effet fortement multiplexé. II existera donc un compromis entre le nombre de lignes adressables et le nombre de niveaux de gris réalisables.

\section{f) Temps de réponse}

Un des paramètres importants pour le confort de l'utilisateur est le temps de réponse de i'écran. Une cellule à CL nématique présente de ce point de vue le comportement décrit figure 15. On distingue :

- un temps de retard $\tau d$, généralement faible,

- un temps de montée $\tau r$ donné par :

$$
\tau \mathrm{r}=\frac{\mathrm{a} \mathrm{d}^{2}}{\varepsilon 0 \Delta \varepsilon\left(\mathrm{V}^{2}-\mathrm{Vs}^{2}\right)}
$$

où $\eta$ est la viscosité du CL et a une constante dépendant de ce dernier.

On remarque que le temps de montée varie comme $1 /\left(\mathrm{V}^{2}-\mathrm{Vs}^{2}\right)$ et pourra donc être réduit par l'application de tensions suffisantes,

- un temps de descente $\tau$ indépendant de la tension, exprimant ainsi le retour à l'équilibre des molécules sous l'action de la seule énergie enmagazinée lors de lapplication préalable d'un champ. $\tau$ f' s'exprime par :

$$
\tau \mathrm{f}=\frac{\mathrm{b \eta d^{2 }}}{\mathrm{n}^{2} \mathrm{k}}
$$

oú b est une constante dépendant des propriétés du CL. On remarquera au passage que $\tau$ r tout comme $\tau$ f varie comme $d^{2}$ ( $d=$ épaisseur de la cellule). L'obtention de dispositifs rapides dépendra donc dans une large mesure de la réalisation de cellules le plus mince possible et souvent d'un compromis entre vitesse et qualité optique. On doit en effet également tenir compte des contraintes sur l'épaisseur liées à l'optimisation du comportement optique des matrices ; une baisse de l'épaisseur se traduisant généralement, pour un CL donné, par une baisse du contraste.

Compte tenu des expressions de $\tau r$ et $\tau$, le temps de réponse d'une matrice s'exprime par :

$$
\begin{aligned}
T r=\tau r+\tau f & \left.=\frac{\eta d^{2}}{\Pi^{2} k}\left(\frac{a}{\left(\frac{V o n}{V o f f}\right)^{2}-1}\right)+b\right) \\
& =\frac{\eta d^{2}}{\Pi^{2} k}(a \sqrt{N}+b)
\end{aligned}
$$


On constate ainsi que le multiplexage tend à dégrader le temps de réponse, celui-ci croissant comme $\sqrt{\mathrm{N}}$ pour un CL donné.

Les temps obtenus actuellement sont de l'ordre de 100 à $200 \mathrm{~ms}$. Un effort de recherche important vise, chez tous les fabricants, à réduire ce temps de façon à le rendre compatible avec l'affichage d'une image vidéo ( $\operatorname{Tr} \leq 40 \mathrm{~ms}$ ).

\section{g) Couplages}

Le couplage, influence d'un motif affiché sur le reste de l'image, reste un des problèmes les plus ardus de l'adressage matriciel. Dans le cas des matrices à CL à multiplexage direct, le couplage provient de l'influence du contenu de l'image sur le terme $\mathrm{Vc}^{2} \frac{\mathrm{N}-1}{\mathrm{~N}}$.

Ce terme constant dans l'idéal, subit des distorsions dues :

- au temps de montée non nul des signaux colonnes (voir figure 16),

- au couplage capacitif lignes/colonnes associé à l'impédance non nulle des drivers lignes.

- etc...

Ces phénomènes dépendent du nombre de transitions observées sur les signaux et par suite des motifs affichés. Les termes en cause sont d'autant plus influents que le nombre de lignes est important, le seuil électrooptique raide et la fréquence trame élevée. On peut essayer d'en diminuer l'importance en :

- minimisant au maximum la résistance interne des alimentations V1 à V5,

- minimisant la résistance de sortie des drivers lignes et colonnes,

- commutant de façon aléatoire la polarité des signaux de commande au cours de la trame : la polarité de chaque ligne étant elle-même inversée toutes les trames. Le but visé est de créer une série de transitions sur les signaux colonnes sans corrélation avec le contenu de l'image, rendant ainsi le terme de "couplage" indépendant de ce dernier.

\section{h) Optimisation des caracteristiques}

Comme on a pu le constater au cours de ce bref tour d'horizon, la conception et l'utilisation d'un matrice de $\mathrm{CL}$ à multiplexage direct implique un compromis entre nombre de lignes de l'écran, nombre de niveaux de gris, vitesse, qualité de l'image... Ce compromis constitue le prix à payer pour la simplicité de réalisation de ces écrans et à justifié l'étude d'autres technologies telles que les technologies à matrices actives qui permettent dans une certaine mesure de relaxer les contraintes. Néanmoins la technologie à multiplexage direct continue à progresser, notamment par la mise au point de mélanges CL sans cesse plus performants, et il semble difficile de pouvoir conclure de façon définitive quant au compromis qui pourrait être obtenu. 


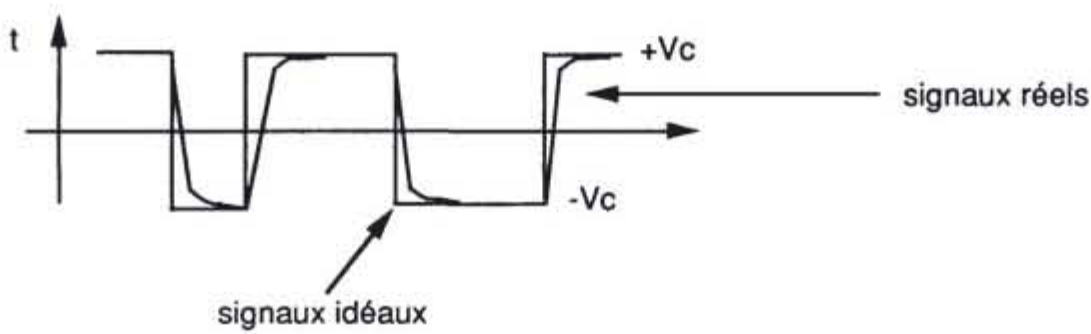

Fig. 16 - Dégradation des signaux colonnes par les temps de montée
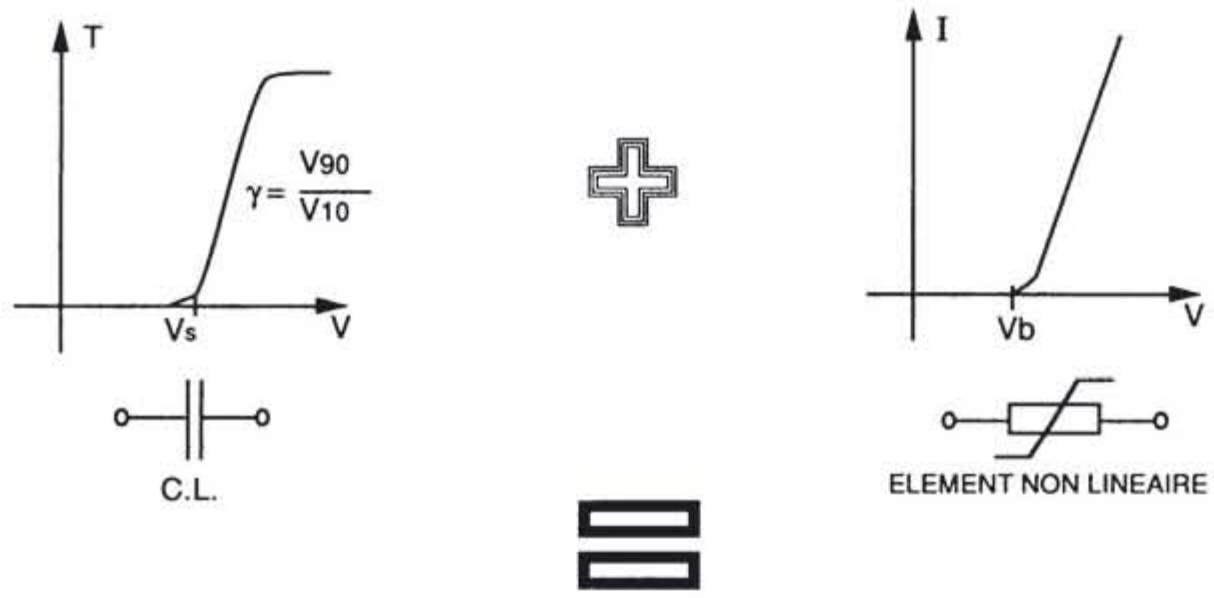

ELEMENT NON LINEAIRE
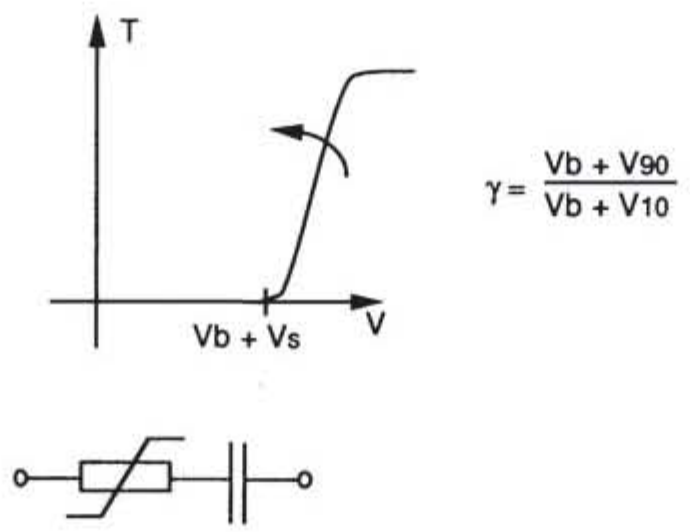

Fig. 17 - "Principe de base" de l'adressage par matrice de dipoles non linéaires 


\section{IV - DES ECRANS A MULTIPLEXAGE DIRECT AUX MATRICES ACTIVES}

\section{a) Introduction}

Comme décrit lors du chapitre précédent, le taux de multiplexage $\mathrm{N}$ est limité par la raideur du seuil électrooptique $\gamma=$ V90N10. Une des solutions proposées pour augmenter le paramètre consiste (voir figure 17) à placer en série avec le pixel un élément non linéaire de tension de seuil Vt.

Le paramètre $\gamma$ résultant de cette association devient alors :

$$
\begin{aligned}
& \gamma=\frac{V t+V 90}{V t+V 10}=\frac{c+\gamma}{c+1} \\
& \text { ou } c=\frac{V t}{V 10} \approx \frac{V t}{V s}
\end{aligned}
$$

ainsi si on place en série un CL de seuil 1,5 V et de $\gamma=1,70$ (donc limité à des taux de multiplexage de 4) et un dispositif non linéaire de seuil $12 \mathrm{~V}$, on obtient un paramètre $\gamma=$ i,08 permettant maintenant un taux de multiplixage de 180.

\section{b) Structure des matrices et composants non linéaires envisageables}

La structure des matrices est décrite figure 18.

Le pixel est maintenant connecté à la ligne de sélection par l'élément non linéaire, la structure des électrodes colonnes restant inchangée.

Différentes technologies ont été étudiées pour réaliser les éléments non linéaires. Parmi les plus courantes (figure 19) on trouve :

- Les diodes MIM (Metal Isolant Metal) qui utilisent les propriétés de conduction non linéaire dans les oxydes (généralement Ta2 O5).

- Les associations de diodes Silicium amorphe (couches minces de silicium déposé par décomposition chimique de silaneș) en série, en anneau ou mixtes.

On notera que l'élément non linéaire doit avoir un comportement symétrique afin de pouvoir adresser le CL selon les 2 polarités.

\section{c) Adressage des matrices actives à dipoles non linéaires}

Si l'on suit le raisonnement qui a présidé à la réalisation des premiers écrans de ce type, ceux-ci s'adressent de la même façon qu'un écran à multiplexage direct. Cependant, si l'on examine en détail le comportement du couple pixel-dipole lors d'une sélection (figure 20 et $20 \mathrm{~b})$, on constate qu'en réalité ce dernier se comporte comme un interrupteur, en effet :

- de to à $t 1$ le pixel est chargé à VI - Vc - Vt

- de 11 à 12 on observe une chute de la tension pixel liée au blocage non instantané de l'élément non linéaire et surtout à la présence d'une capacité parasite Cp à ses bornes.

- Après t2 (en dessous du seuil), le dipole ne conduit plus et la tension pixel reste constante jusqu'à la trame suivante si l'on néglige les fuites dues au $C L$ ( $R C=50$ à 100 ms ou mieux) et à la conduction résiduelle du dipole non linéaire. 


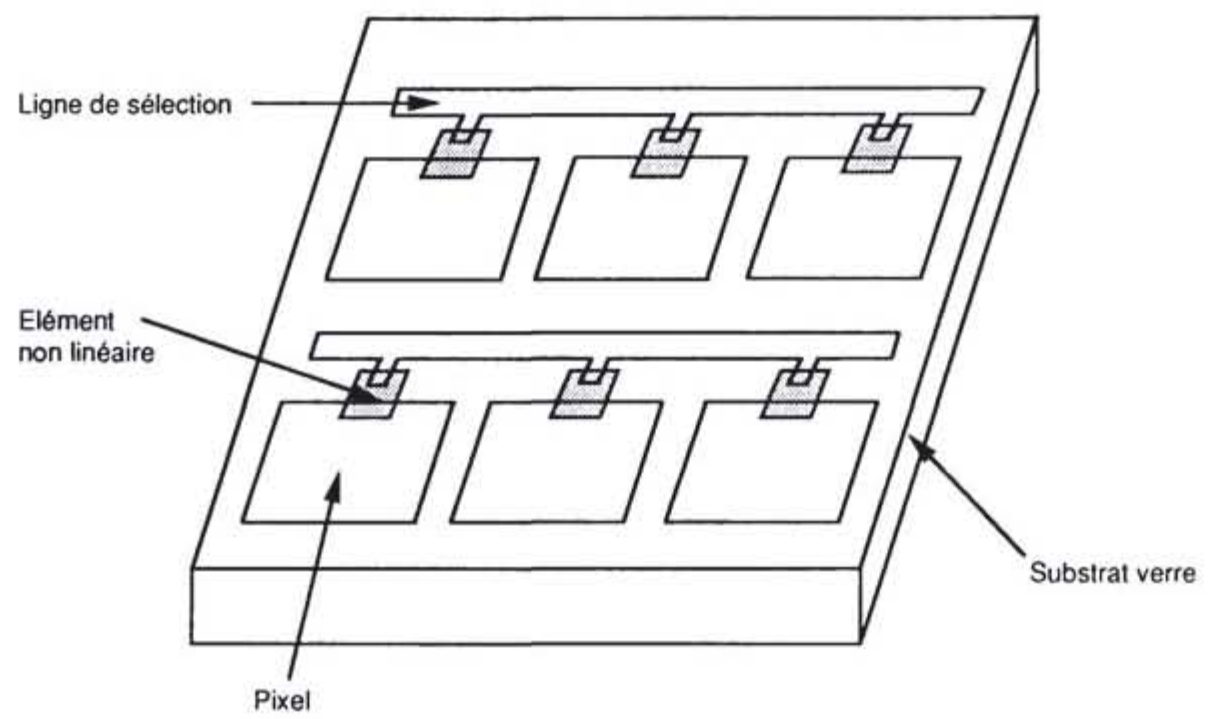

Fig. 18 - Structure des substrats lignes dans une matrice active à dipoles

- DIODES SILICIUM AMORPHE

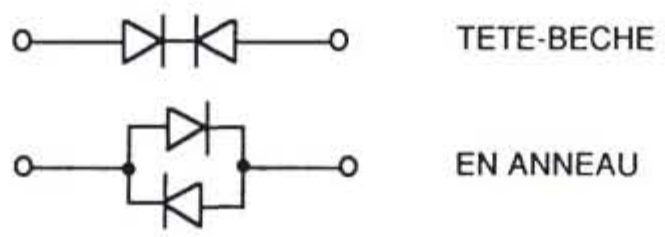
$\circ$
$\circ$
$\circ$

- MIM METAL-ISOLANT-METAL

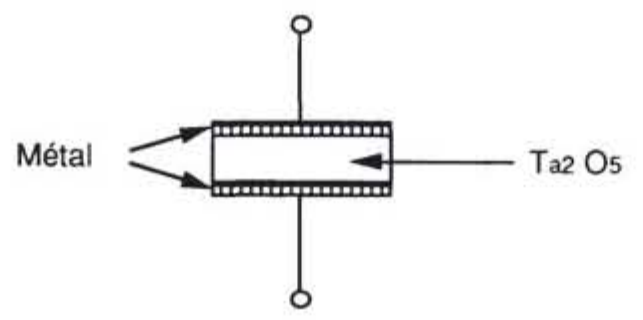

Fig. 19 - Dipoles non linéaires 


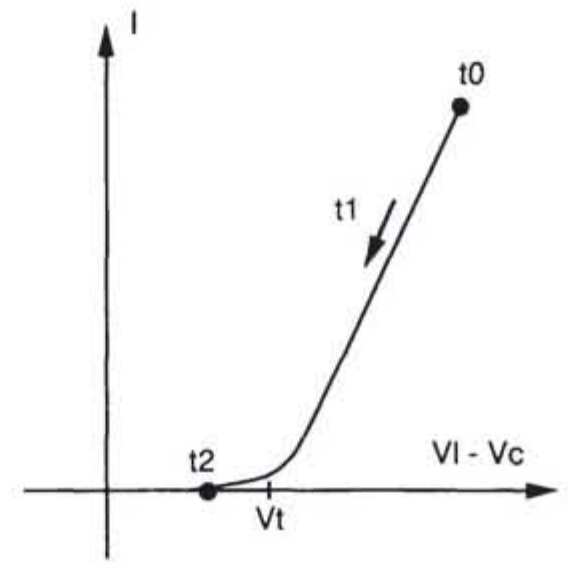

Fig. 20 a - Point de fonctionnement du dipole non linéaire

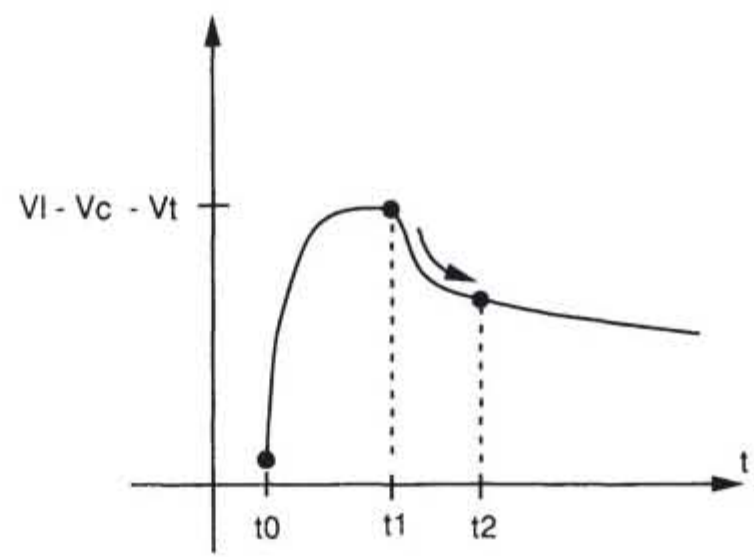

Fig. 20 b - Tension pixel lors d'un sélection 
Tout se passe donc pour le CL comme si le taux de multiplexage était de 1 . La structure décrite permet ainsi de lever les contraintes liées au multiplexage, le nombre de lignes adressables n'étant plus limité que par le temps $\mathrm{t} 1$ - to nécessaire à la charge d'un pixel ( 20 us donc 1000 lignes peuvent être adressées par trame de 20 ms).

\section{d) Des matrices actives a dipole aux matrices de transistors}

L'introduction de dipoles non linéaires dans la matrice relaxe les contraintes imposées au $\mathrm{CL}$ par le multiplexage direct . II est ainsi plus aisé de réaliser des écrans rapides et à fort contraste. Cependant, l'obtention de niveaux de gris reste délicate. En effet le dipole non linéaire rempli à la fois les rôles d'élément de commutation et d'amenée du signal, ainsi la tension réellement appliquée au pixel $(\mathrm{VI}-\mathrm{Vc}-\mathrm{Vt})$, dépendra de ces caractéristiques.

En conséquence toute dispersion sur la valeur de Vt sera répercutée sur l'image. Les matrices actives à transistors permettent, comme nous allons le voir, de résoudre ce type de problèmes.

\section{V - MATRICES ACTIVES A TRANSISTORS}

\section{a) Structure de la matrice et des transistors}

Les transistors utilisés dans les matrices actives sont les analogues pour les semi conducteurs en couches minces du transistor MOS pour le Silicium cristallin. Ces composants (voir figure 21) sont obtenus par dépôt et gravure de couches métalliques, isolantes et semi conductrices. Ces dernières sont le plus souvent réalisées en silicium amorphe hydrogéné, les composants réalisés avec ce matériau présentant des caractéristiques (voir figure 22) qui comme nons le verrons sont suffisantes pour l'adressage d'une matrice à CL. Dans une telle matrice (voir figure 23) le transistor est relié par sa "source" au pixel, par son "drain" a une colonne de l'écran et par sa grille à une ligne. Dans cette structure, il est à noter que lignes, colonnes, pixels et transistors sont sur le même substrat ; le second substrat, quant à lui, supporte une électrode de référence (commune à tous les pixels et contituant la seconde armature du condensateur) portée au potentiel Vref.

Comme indiqué figure 24 , on rencontre également une seconde structure, dite "Bus non croisés", où en déplaçant la position de la capacité pixel dans le circuit, on peut placer lignes et colonnes sur chacun des substrats, évitant tout risque de court-circuit lignes/colonnes.

\section{b) adressage}

Les transistors couches minces (TCM) utilisés dans les matrices sont bloquants $(R=R$ off) quand une tension négative ou faible est appliquée entre grille et source ou drain (NB : les 2 électrodes n'ont pas du fait de l'absence de substrat les rôles distincts que l'on peut leur voir attribué dans la technologie MOS classique). Ils deviennent conducteurs $(R=R o n)$ lorsque la tension appliquée entre grille et source ou drain dépasse une valeur de seuil Vt à partir de laquelle un canal de conduction se crée par apport d'électrons à l'interface Isolant/Semi conducteur. Ils se comportent donc comme des interrupteurs commandés par la grille, permettant ainsi la charge des pixels à la tension colonne lorsque les lignes sont sélectionnées et le maintien de cette charge le reste du temps. Le mode d'adressage qui en découle est indiqué figure 25. Afin d'obtenir une conduction et un blocage correct des transistors, VI et Vc doivent respecter : 


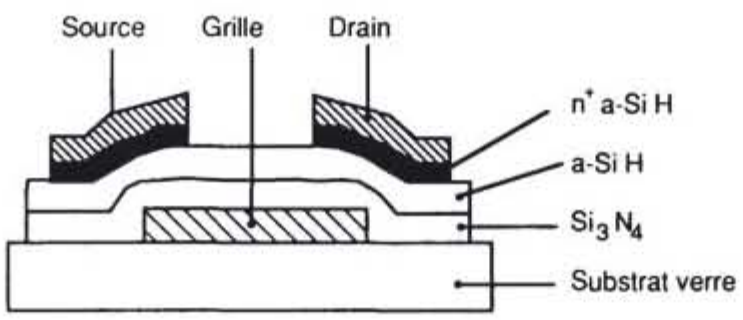

Fig. 21 - Structure d'un transistor couches minces (TCM)

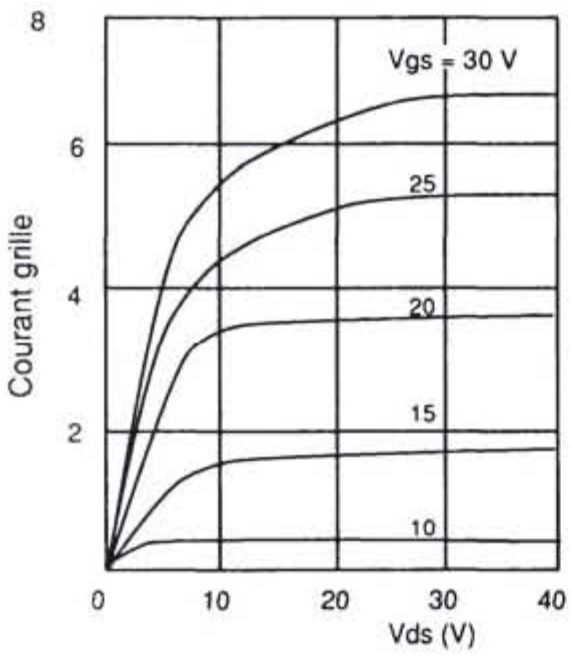

Tension Drain Source

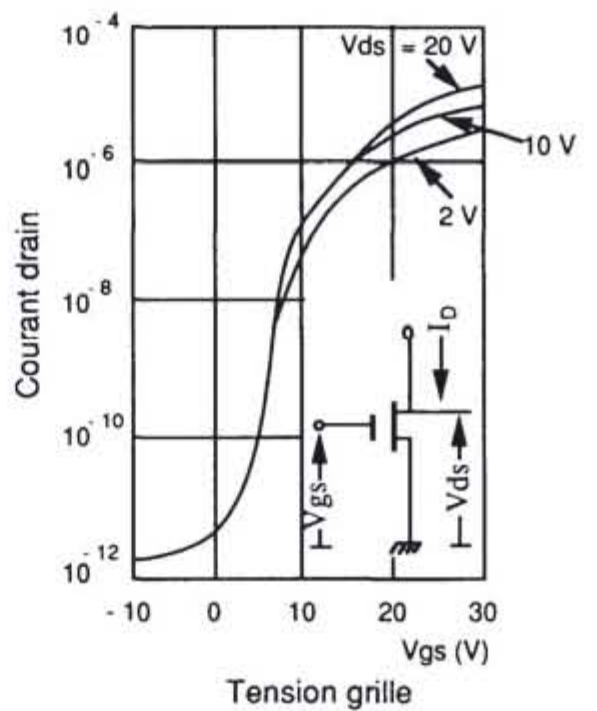

Fig. 22 - Caractéristiques ld (Vds) et Id (Vgs) d'un TCM à base de silicium amorphe hydrogéné 

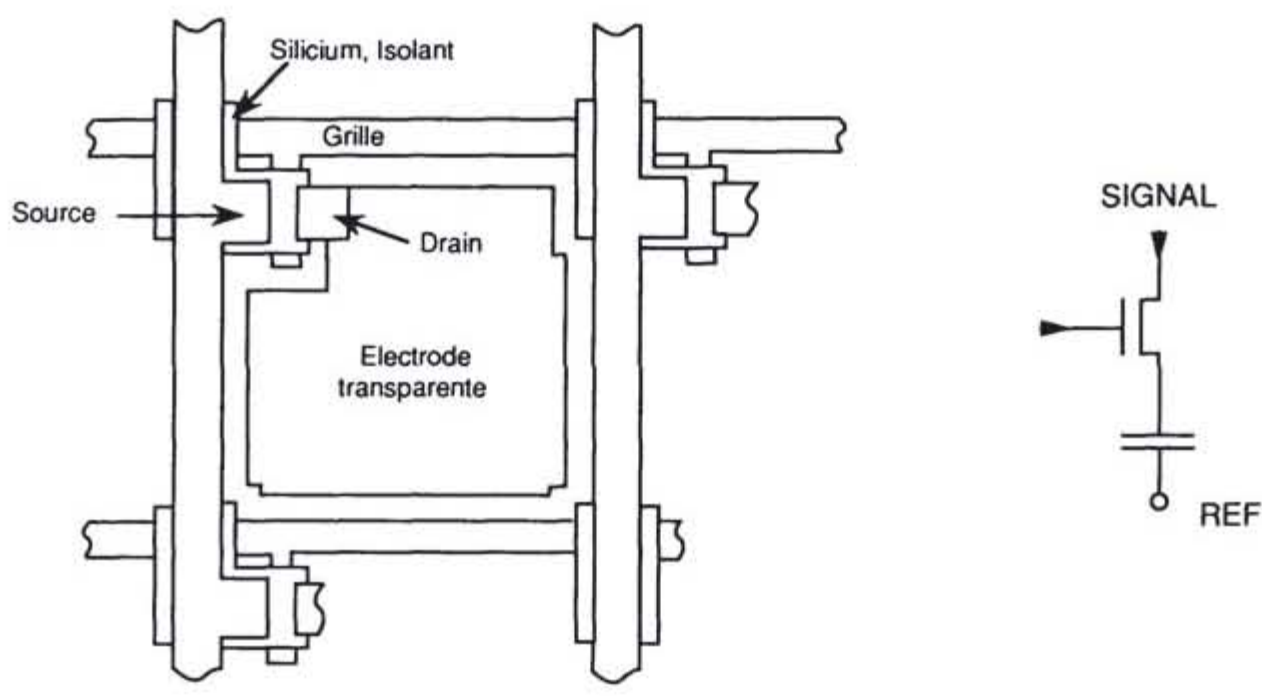

Fig. 23 - Structure d'un pixel d'une matrice active à transistors
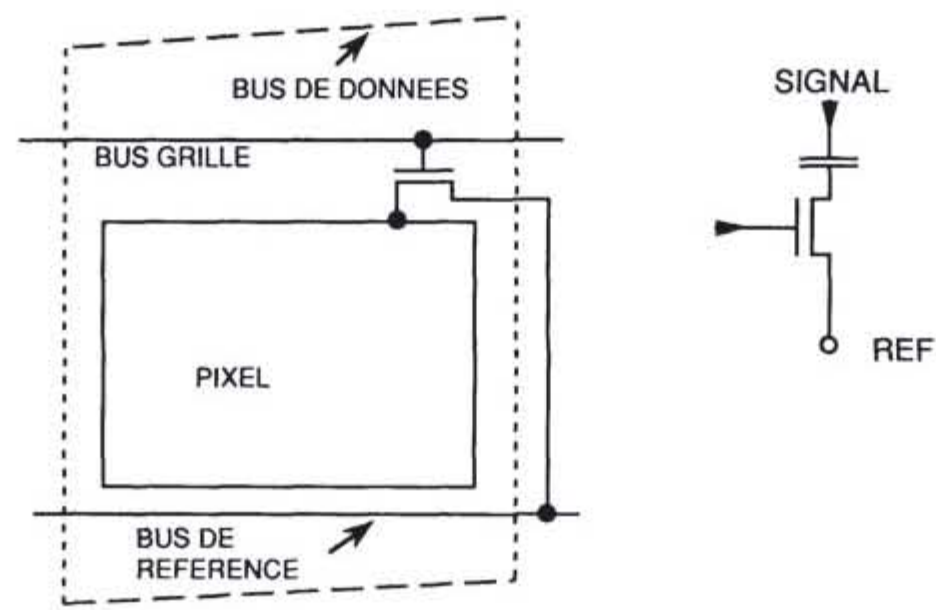

Fig. 24 - Structure "Bus non croisés" 

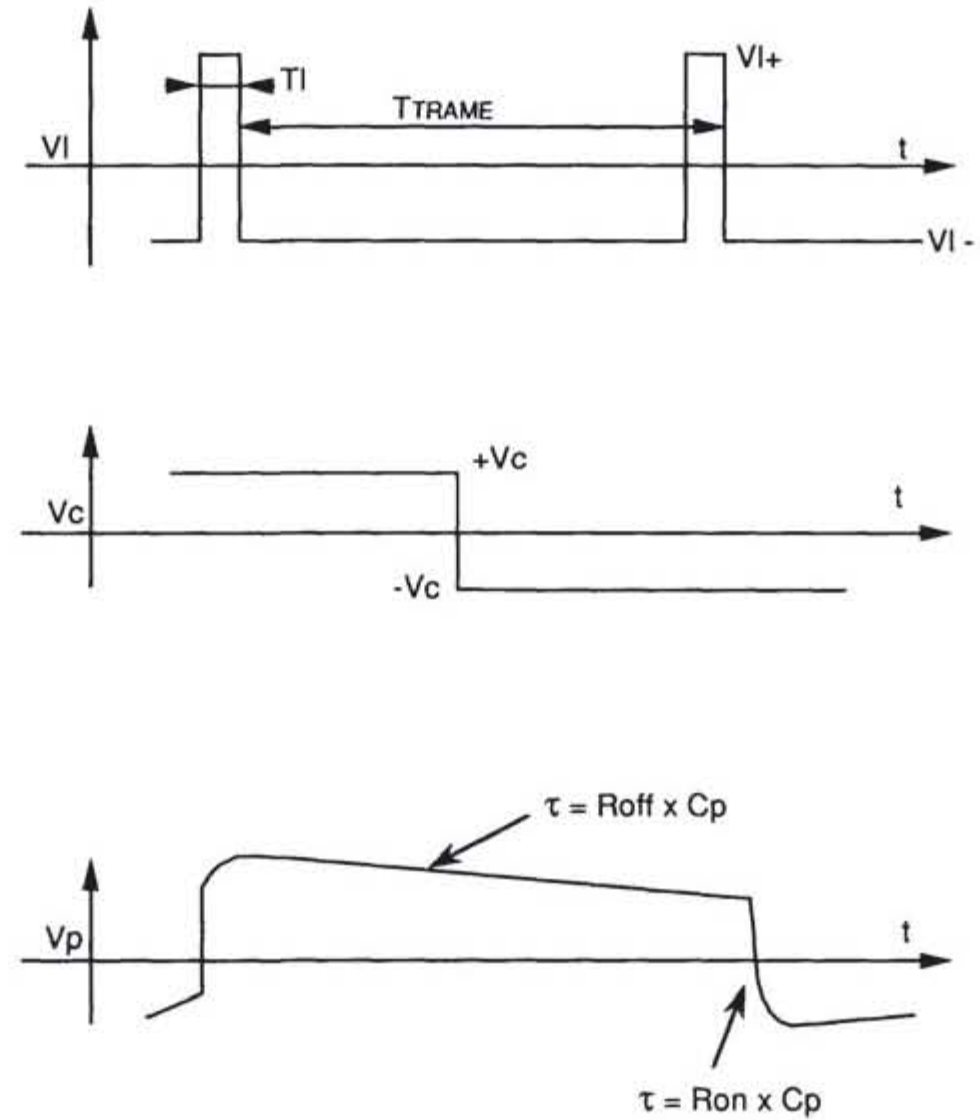

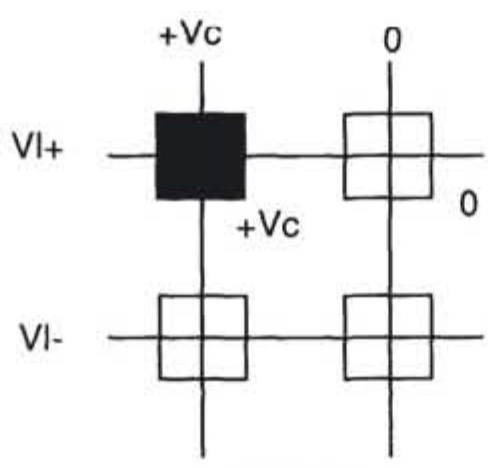

TRAME K

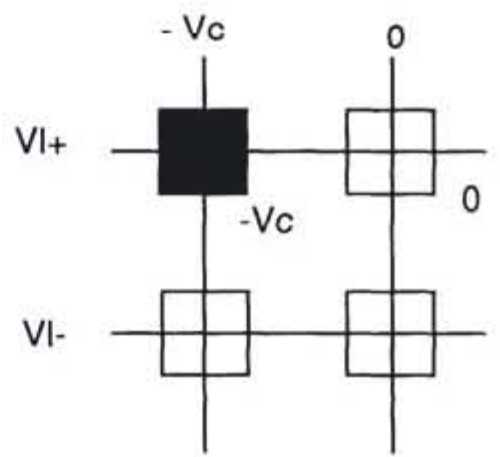

TRAME $\mathrm{K}+1$

Fig. 25 - Adressage d'une matrice active 
$\mathrm{Vl}+\geq \mathrm{Vc}+\mathrm{Vt}$

et $\mathrm{VI}-\leq-\mathrm{V} c+\mathrm{Vt}$

Les performances des transistors doivent par ailleurs être telles que :

Ron $\mathrm{Cp} \leq$ TLIGNE ( $\approx 60 \mu \mathrm{s}$ ) charge du pixel

et Roff $\mathrm{Cp} \gg$ TTRAME ( $\approx 20 \mathrm{~ms}$ ) maintien de la charge

où $\mathrm{C} p$ représente la capacité pixel $(\approx 0,5 \mathrm{pF})$

d'où $\frac{\text { Roff }}{\text { Ron }}>10^{4}$ à $10^{5}$

et Ron < qq $10 \mathrm{M} \Omega$.

Ces performances restent compatibles (voir figure 21) avec l'utilisation de TCM Silicium Amorphe.

On notera enfin que, si les conditions ci-dessus sont remplies, la tension appliquée aux pixels ne dépend que de la tension colonne lors de la sélection et reste très peu sensible aux dispersions de caractéristiques des TCMs de la matrice.

\section{c) Phénomenes parasites, couplage}

Comme dans tout sytème, la présence d'éléments parasites tend à en dégrader le fonctionnement. Le comportement des matrices actives étant essentiellement capacitif on comprendra aisément que toute capacité parasite introduite dans le circuit tende à en altérer les performances. On distingue (en 1ère approximation) 2 familles de capacités parasites aux rôles distincts (voir figure 26) :

- celle liée au transistor, la capacité grille $\mathrm{Cg}$,

- celle liée aux capacités pixel colonnes Cpc.

\section{1 - Influence de la capacité grille $\mathrm{Cg}$}

Comme décrit précédemment; le passage à l'état conducteur d'un TCM s'accompagne d'une caccumulation de porteurs à l'interface Isolant/SC. Lors de la charge d'un pixel, la charge accumulée est donnée par :

$$
Q=C g(V I+ \pm V c-V t)
$$

En fin de charge, la tension drain-source tendant à s'annuler, la charge Q s'évacue pour moitié par la colonne et pour moitié vers le pixel (voir figure 27). L'effet résultant peut être aisément calculé en exprimant la conservation de la charge sur l'armature libre du pixel. Ainsi :

$$
\begin{aligned}
& \mathrm{Q}=\mathrm{CpVc}-\frac{\mathrm{Cg}}{2}(\mathrm{VI}++\mathrm{Vc}-\mathrm{Vt})=\mathrm{CpVc^{ \prime }} \\
& \text { d'où } V c^{\prime}=\left(1-\frac{\mathrm{Cg}}{2 \mathrm{Cp}}\right) V c-\frac{\mathrm{Cg}}{2 \mathrm{Cp}}\left(V l_{+}-V \mathrm{~V} t\right)
\end{aligned}
$$

La présence de $\mathrm{Cg}$ se traduit donc par l'apparition d'une composante continue sur le $\mathrm{CL}$ et un gain inférieur à 1 sur les signaux colonnes appliqués. Sachant que $\mathrm{Cg}=50$ à $100 \mathrm{fF}, \mathrm{Cp}$ $\approx 0,5 \mathrm{pF}$ et $\mathrm{VI}+-\mathrm{Vt}=5$ à $10 \mathrm{~V}$, l'amplitude de la composante continue peut atteindre quelques volts : elle devra donc être impérativement compensée par l'application d'une tension de décalage sur l'électrode de référence de la matrice. 


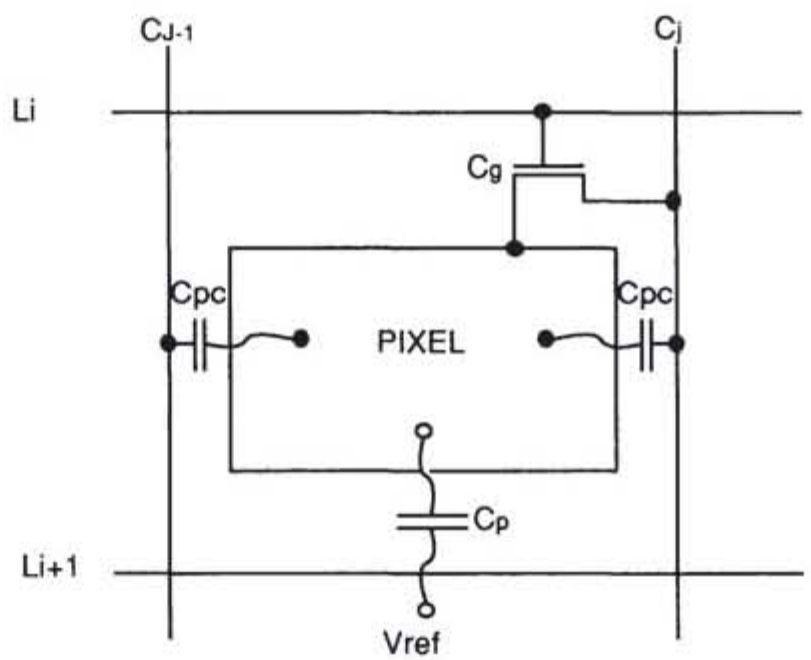

Fig. 26 - Eléments parasites d'un pixel d'une matrice

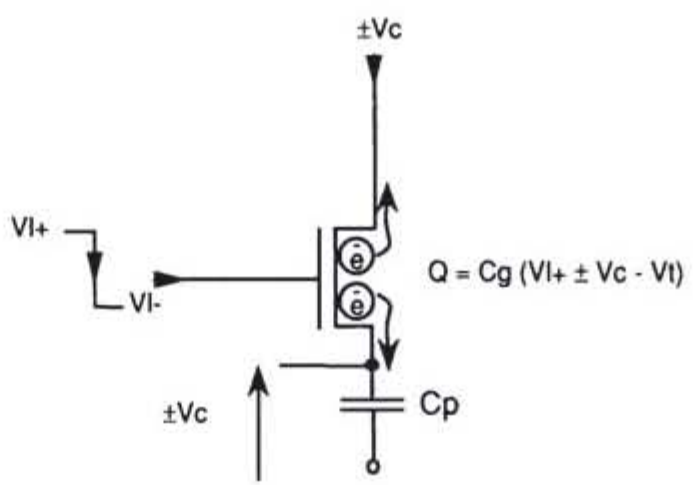

Fig. 27 - Influence de la capacité grille lors du bloquage du TCM 

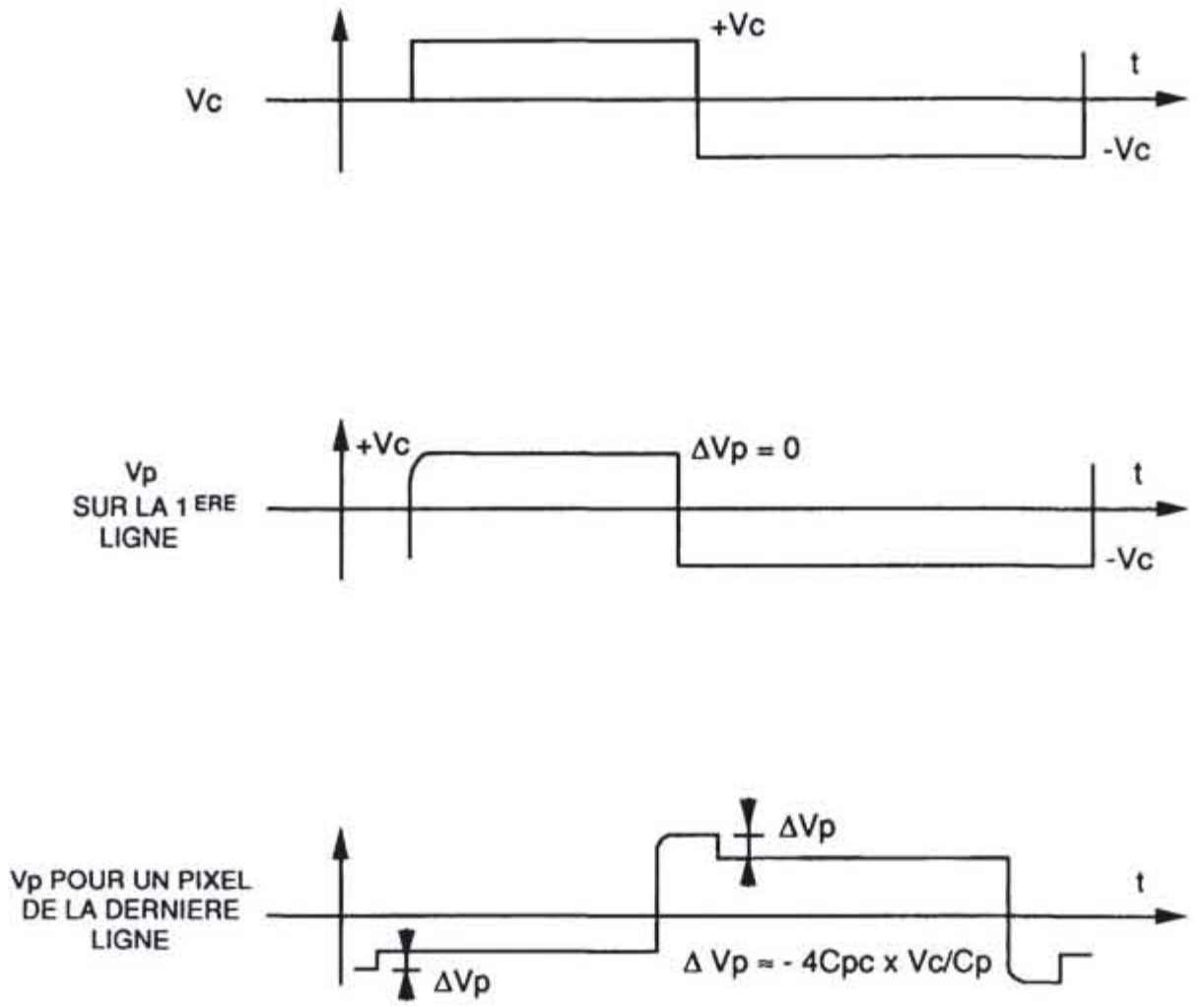

Fig. 28 - Influence des capacités parasites pixel-colonne sur la tension pixel Vp 


\section{2 - Influence de capacités Coc}

Ces capacités interviennent durant toute la durée de la trame, elles forment en effet avec la capacité pixel $C_{p}$ un pont diviseur qui répercute sur le pixel dans le rapport $\frac{2 C p c}{C p}$ toute variation des tensions colonnes. Le couplage qui en résulte peut, si l'on n'y prend garde, altérer de façon importante l'image affichée. En effet, considérons par exemple l'affichage d'une zone uniforme (figure 28) et le comportement dans ce cas de la tension pixel pour les premières et dernières lignes de l'écran :

- pour les premières lignes, aucune variation donc aucune influence de la tension colonne n'est enregistrée au cours de la trame $(\Delta V p=0)$,

- à l'opposé, une excursion de 2 Vc est appliquée aux colonnes peu de temps après l'adressage des dernières lignes. La tension appliquée sur les pixels de ces dernières sera donc réduite de $\frac{4 C p c}{C p} V_{c}(=10 \%$ Vc).

Le couplage induirait donc dans ce cas une dissymétrie haut/bas dans la réponse de la matrice. II est heureusement possible d'y remédier en alternant la polarité des signaux colonnes ligne à ligne et trame à trame, évitant ainsi de particulariser le comportement des différentes zones de l'écran. Mieux, on démontre que cette méthode, efficace dans le cas ci-dessus, l'est quelque soit l'image affichée. Elle permet, de plus, de limiter le papillotement (flicker) des images.

\section{VI - CONCLUSION}

Comme on a pu le constater l'utilisation de matrices actives nécessite, pour en tirer le meilleur parti, de soigner l'adressage et de connaître au mieux leur comportement. Les performances obtenues sont alors à la hauteur des prouesses technologiques qu'exige leur fabrication. Contraste, angle de vue et vitesse des écrans sont en effet nettement supérieurs à ceux des écrans à multiplexage direct en vente actuellement. Cependant les difficultés de fabrication de tels composants (un million de transistors sur quelques $\mathrm{dm}^{2}$ ) limitent à l'heure actuelle ceux-ci à des tailles modestes (5 à 6 "); le marché des écrans de grande taille restant couvert par des technologies classiques.

Pour ces raisons et tenant compte de tous les arguments présentés lors de cet exposé, il s'avère difficile de pronostiquer dès maintenant la suprématie future de telle ou telle technologie. Plus raisonnablement, il semble que l'équipementier aura à mettre en balance ses impératifs de coût et de performances pour faire son choix. 


\section{BIBLIOGRAPHIE}

Pour de plus amples informations sur l'adressage et pour approfondir les nombreux problèmes qui n'ont pu être abordés, le lecteur pourra consulter :

- B. BAHADUR : "Liquid Crystal Displays", Molecular Crystals and Liquid Crystals, Vol. 109(1), (1984).

- B. MOUREY ET AL : "Ecrans à cristal liquide", Revue Technique THOMSON CSF, Vol. 18(4), (1986).

- E. KANEKO : "Liquid Crystal TV display", ADOP, KTK Scientific Publishers / TOKYO, (1987). 